\title{
Bag3 P209L myopathies and efficacy of blocking signaling pathways with the therapeutic peptide, MMI-0100
}

\author{
Ashley R. Hacker ${ }^{1}$, Jessica M. Berthiaume ${ }^{2}$, Monte S. Willis ${ }^{2,3}$ \\ ${ }^{1}$ Indiana University School of Medicine, ${ }^{2}$ Department of Pathology \& Laboratory \\ Medicine, Center for Musculoskeletal Health, ${ }^{3}$ Section of Cardiology, Department \\ of Internal Medicine, Indiana University, Indianapolis, IN \\ Background and Hypothesis: The Bcl2-associated anthanogene (BAG) 3 \\ protein is a member of the BAG family of cochaperones, which play a critical role \\ in cellular processes, including protein degradation and turnover. Over 30 Bag3 \\ mutations have been identified, including a Proline209Leucine (P209L) missense \\ mutation which causes a severe childhood cardiomyopathy. The mechanism by \\ which Bag3 mutations causes cardiomyopathy is currently unknown, but the p38/ \\ MAPK signaling cascade has been shown to be altered in our animal model that \\ coexpresses the human Bag3 P209L gene. We hypothesized the cell-permeant \\ peptide, MMI-0100, which is known to inhibit MAPK-activated protein kinase 2 \\ (MK2) activity in the p38/MAPK signaling cascade, would alleviate or reduce \\ cardiac dysfunction.
}

Experimental Design: Echocardiographic analysis of cardiac function of cardiacspecific Bag3 P209L transgenic (and wildtype littermate control) mice (20 - 22 months of age) was assessed by high-resolution ultrasound echocardiography (VisualSonics Vevo 2100, MS550D probe, cardiology package) to document the established disease-related cardiac dysfunction at baseline. Mice were then treated with $100 \mu \mathrm{g} / \mathrm{kg} /$ day MMI-0100 nebulized daily for 30 days. Follow-up echocardiography was performed at 10, 20, and 30 days of MMI-0100 treatment. Echocardiographic analysis was performed to determine the systolic function (EF $\%, \mathrm{FS} \%$ ), chamber dimensions, and wall thickness in systole and diastole using Vevo 2100 Workstation software package.

Results: Blinded analysis of echocardiographic data identified that Bag3 P209L $\mathrm{Tg}+$ mice had a baseline cardiac dysfunction compared to wildtype controls at 20 months of age (WT $=76 \% \mathrm{EF}, 44 \% \mathrm{FS} ; \mathrm{Tg}+=66 \% \mathrm{EF}, 36 \% \mathrm{FS}$ ). MMI-0100 treatment significantly attenuated this dysfunction by 20 days of MMI-0100 treatment (WT $=79 \% \mathrm{EF}, 47 \% \mathrm{FS}$; $\mathrm{Tg}+=80 \% \mathrm{EF}, 48 \% \mathrm{FS}$ ), consistent with demonstrating for the first time MK2's role in mediating p38 signaling in the pathogenesis of Bag3 P209L cardiomyopathy.

Conclusion and Potential Impact: The MMI-0100 peptide has proven efficacious in several animal models of fibrosis driven by p38 signaling, as MK2 is a p38 downstream mediator. Future studies seek to translate the use of the MMI-0100 peptide in pediatric patients with Bag3 P209L cardiomyopathy through compassionate use FDA pathways. 